\title{
HARMONY or discord in cardiovascular outcome trials of GLP-1 receptor agonists?
}

Marion Mafham ${ }^{1}$, David Preiss ${ }^{1}$

${ }^{1}$ MRC Population Health Research Unit, Clinical Trial Service Unit, Nuffield Department of Population Health, University of Oxford, Oxford, UK

Correspondence to:

Dr David Preiss

MRC PHRU, CTSU, Richard Doll Building, Roosevelt Drive, Oxford, OX3 7LF, UK

Phone: +44 1865743527

Email: david.preiss@ndph.ox.ac.uk

Word count: 767

Disclosures: CTSU has a policy of not accepting any personal payments directly or indirectly from industry. DP reports being an investigator on a trial of a glucose-lowering agent funded by a grant to the University of Oxford by Boehringer Ingelheim, but he receives no personal payment. 
In 2017, GlaxoSmithKline announced its intention to withdraw the GLP-1 receptor agonist, albiglutide, for commercial reasons prior to the emergence of definitive evidence regarding its cardiovascular safety and efficacy. In this issue, Hernandez and coinvestigators report results from the 9,463 participant HARMONY trial, demonstrating that albiglutide has beneficial effects on cardiovascular outcomes when given to people with type 2 diabetes and atherosclerotic cardiovascular disease. ${ }^{1}$ Over a median follow-up of 1.6 years, allocation to albiglutide, given weekly by subcutaneous injection, produced a proportional $22 \%$ (95\% confidence interval $[\mathrm{Cl}] 10 \%$ to $32 \%$ ) reduction in the risk of the primary outcome (cardiovascular death, myocardial infarction or stroke) compared to placebo when added to standard care. This clear result is an important step in the emerging story of GLP-1 receptor agonists.

The HARMONY results strengthen the case for the use of GLP-1 inhibitors, as a class, in patients with atherosclerotic cardiovascular disease though, as Hernandez et al suggest, results from the five randomized outcome trials of various GLP-1 receptor agonists completed to date have been inconsistent. Is this inconsistency due to differences in the properties and effects of the different drugs, or is it instead due to differences in trial design and conduct? The LEADER trial of daily liraglutide ${ }^{2}$ and the SUSTAIN $-6^{3}$ trial of weekly semaglutide studied people with diabetes and either cardiovascular disease or increased cardiovascular risk, and both showed statistically significant reductions in cardiovascular events. The proportional reductions in the composite of cardiovascular death, non-fatal myocardial infarction or non-fatal stroke were $13 \%$ (95\% Cl 3\% to $22 \%$ ) and $26 \%$ (95\% Cl 5\% to $62 \%$ ) respectively, broadly consistent with HARMONY. The EXSCEL trial, in a similar population, failed to convincingly demonstrate cardiovascular benefit of weekly exenatide with a non-significant $9 \%(95 \% \mathrm{Cl} 0 \%$ to $17 \%)$ proportional reduction in the same primary outcome, but reported adherence was poor. ${ }^{4}$ The ELIXA trial of daily lixisenatide in acute coronary syndrome patients showed no effect of lixisenatide on a slightly broader primary outcome. ${ }^{5}$ Albiglutide, liraglutide and semaglutide partly share a common molecular structure in that their GLP-1 receptor ligands are based on human GLP-1. By contrast, exenitide and lixisenetide are exendin-4 based. Whether these structural differences translate into clinically relevant differences in treatment effects, however, is not clear. Small head-to-head trials do not suggest an obvious link between the different structures of these 
drugs and their impact on established cardiovascular risk factors, although the GLP-1 based drugs may produce slightly larger reductions in HbA1c. ${ }^{6}$ However, the diminishing effect on HbA1c over time observed in ELIXA and EXSCEL may reflect poor adherence potentially accounting for the apparently discrepant results from these studies.

These trials were relatively short with mean follow-up of 2 years or less in HARMONY, ELIXA and SUSTAIN-6, and around 3.5 years in LEADER and EXSCEL. This may complicate a trial's ability to generate reliable results because harms can appear late and because benefits, as with cholesterol lowering, can emerge after a lag period from the start of treatment. ${ }^{7}$

The mechanism or mechanisms by which albiglutide, and other GLP-1 receptor agonists, reduce atherosclerotic cardiovascular events is not clear. Modest reductions in $\mathrm{HbA} 1 \mathrm{c}$ of $0.3 \%$ to $0.8 \%$ for 1 to 3 years are unlikely to account for the reduction in risk, and the effect on blood pressure is modest. Furthermore, in the HARMONY trial, differences between treatment arms in the use of insulin and other treatments were not substantial. Effects of GLP-1 receptor agonists on myocardial and arterial function, and on lipid metabolism have also been demonstrated. ${ }^{8}$

International guidelines should reflect the increasing weight of evidence supporting the use of GLP-1 receptor agonists in patients with diabetes and cardiovascular disease. Further studies investigating their effects in people with diabetes but without known vascular disease are now a priority to better inform the clinical community about the selection of glucose-lowering drugs in such patients. These studies will require highly streamlined, innovative methods to produce robust results cost-effectively. They should have sufficiently long duration of follow-up to assess emerging benefits and hazards reliably, but must ensure good adherence to treatment, for example by considering an active run-in phase. Since the beneficial cardiovascular effects of these drugs may not be mediated through control of glycaemia, clinical trials of GLP-1 receptor agonists in people with cardiovascular disease but without diabetes are warranted and such trials are underway. ${ }^{9}$ Of note, substantial weight loss may be achieved in obese non-diabetic individuals treated with high doses of some GLP1 receptor agonists, supporting the potential utility of these drugs in populations without diabetes. ${ }^{10}$ Finally, given the clear cardiovascular benefit observed with albiglutide in HARMONY, GlaxoSmithKline should reconsider making it available to patients. 


\section{References}

1. Hernandez AF, Green JB, Janmohamed S, D'Agostino RB, Granger CG, Jones NP, et al. Albiglutide and Cardiovascular Outcomes in Patients with Type 2 Diabetes and Cardiovascular Disease. The Lancet. 2018; X(X): X.

2. Marso SP, Daniels GH, Brown-Frandsen K, Kristensen P, Mann JF, Nauck MA, et al. Liraglutide and Cardiovascular Outcomes in Type 2 Diabetes. New Eng J Med. 2016; 375(4): 311-22.

3. Marso SP, Bain SC, Consoli A, Eliaschewitz FG, Jodar E, Leiter LA, et al. Semaglutide and Cardiovascular Outcomes in Patients with Type 2 Diabetes. New Eng J Med. 2016; 375(19): 1834-44.

4. Holman RR, Bethel MA, Hernandez AF. Once-Weekly Exenatide and Cardiovascular Outcomes in Type 2 Diabetes. New Eng J Med. 2017; 377(25): 2502.

5. Pfeffer MA, Claggett B, Diaz R, Dickstein K, Gerstein HC, Kober LV, et al. Lixisenatide in Patients with Type 2 Diabetes and Acute Coronary Syndrome. New Eng J Med. 2015; 373(23): 224757.

6. Dalsgaard NB, Vilsboll T, Knop FK. Effects of glucagon-like peptide-1 receptor agonists on cardiovascular risk factors: A narrative review of head-to-head comparisons. Diabetes, obesity \& metabolism. 2018; 20(3): 508-19.

7. The Cholesterol Treatment Trialists Collaboration. Efficacy and safety of cholesterol-lowering treatment: prospective meta-analysis of data from 90,056 participants in 14 randomised trials of statins. Lancet. 2005; 366(9493): 1267-78.

8. Drucker DJ. The Ascending GLP-1 Road From Clinical Safety to Reduction of Cardiovascular Complications. Diabetes. 2018; 67(9): 1710-9.

9. $\quad$ https://clinicaltrials.gov/ct2/show/NCT03574597. Accessed 17 Sept 2018.

10. O'Neil PM, Birkenfeld AL, McGowan B, Mosenzon O, Pedersen SD, Wharton S, et al. Efficacy and safety of semaglutide compared with liraglutide and placebo for weight loss in patients with obesity: a randomised, double-blind, placebo and active controlled, dose-ranging, phase 2 trial. Lancet. 2018; 392(10148): 637-49. 